\title{
Fabrication of CdS/PMMA core/shell nanoparticles by dispersion mediated interfacial polymerization
}

\author{
Jyongsik Jang, * Sunhee Kim and Kyung Jin Lee \\ Received (in Cambridge, UK) 12th March 2007, Accepted 2nd April 2007 \\ First published as an Advance Article on the web 17th April 2007 \\ DOI: $10.1039 / b 703717$ a
}

CdS/PMMA core/shell nanoparticles were synthesized using dispersion-mediated interfacial polymerization, and the transparent PMMA shell not only maintained the optical properties of $\mathrm{CdS}$ core but effectively protected the CdS core from environmental perturbation.

Highly luminescent II-VI semiconductor nanoparticles (NPs) have been extensively studied for the past two decades. ${ }^{1}$ Due to the quantum confinement effect, ${ }^{2}$ nanocrystals show unique sizedependent physical, chemical and optical properties, which are different from the bulk materials. ${ }^{3}$ In addition, II-IV semiconductor NPs have attracted much attention because of their various applications in areas such as biological labeling, light-emitting diodes, single-electron transistors, solar cells, photocatalysts and photonic crystals. ${ }^{4}$

High-quality CdS NPs synthesized by the high-temperature thermal decomposition of precursors exhibit narrow size distribution and excellent luminescent property. ${ }^{5}$ However, the problems in this synthetic method lie in the aggregation and degradation under the atmosphere when the organic solvent (capping agent) is removed. ${ }^{6}$ Compared with CdS NPs, CdS/polymer core/shell NPs provide a unique combination of improved thermal stability and photostability as well as maintaining enhanced dispersity after the removal of organic ligand or the capping agent. ${ }^{7}$ In addition, polymer-coated inorganic nanoparticles have several advantages over the single-component materials from the viewpoint of their potential applications. The uniform coating of organic polymers onto inorganic particles can endow the raw materials with beneficial electrical, optical and catalytic functions. In conventional solution-based methods, there have been found difficulties in controlling the coating of polymer layers on the nanocrystals without aggregation at the nanoscale. Recently, extensive studies have been performed for the encapsulation of II-IV semiconductor NPs with various polymers, which include emulsion polymerization, atomic transfer radical polymerization, layer-by-layer selfassembly of polyelectrolyte, and graft polymerization on the particle surface. ${ }^{8}$ However, these methods have some intrinsic problems such as intricate synthetic procedures and high cost. Therefore, it is still a challenging task to develop facile and novel methods to fabricate nanoparticle/polymer core/shell structures.

Herein, we describe the fabrication of CdS/poly(methyl methacrylate) (PMMA) core/shell nanoparticles using dispersionmediated interfacial polymerization (DMIP). This work represents

Hyperstructured Organic Materials Research Center and School of Chemical and Biological Engineering, Seoul National University, Shinlimdong 56-1, Seoul, 151-742, Korea.

E-mail: jsjang@plaza.snu.ac.kr; Fax: 822888 1604; Tel: 8228807069 the novel demonstration of luminescent NP-embedded polymer nanoparticles synthesis without any nanoparticle aggregation using charge-charge interactions between the CdS surface and initiator. PMMA was employed due to its optical transparency. In addition, the PMMA shells played a role in protecting the surface of $\mathrm{CdS}$ cores from oxidation in the environmental conditions. Our synthetic methodology includes a number of advantages over the other solution-based techniques, such as the formation of polymer layers with uniform thickness, high yield of the product, and simple fabrication steps.

The overall synthetic procedure of PMMA-coated CdS NPs is illustrated in Scheme 1. First, spherical CdS nanoparticles were synthesized from the reaction of $\mathrm{CdCl}_{2}$ and sulfur powder in oleylamine. The molar ratio of cadmium to sulfur was $1: 1 . \mathrm{CdCl}_{2}$ $\left(1 \times 10^{-3} \mathrm{~mol}\right)$ was mixed with oleylamine $(10 \mathrm{~mL})$ and then the temperature was raised to $160{ }^{\circ} \mathrm{C}$. Subsequently, sulfur powder dissolved in oleylamine $(5 \mathrm{~mL})$ was added dropwise to the hot solution. The mixture was kept at $160{ }^{\circ} \mathrm{C}$ for $20 \mathrm{~h}$ under vigorous stirring. Afterwards, the solution was cooled to room temperature, and the resulting $\mathrm{CdS}$ nanoparticles were washed with ethanol to remove any residual precursor. Although most of oleylamine is removed by this procedure, oleylamine remains on the surface of CdS NPs, exclusively. Therefore, the particle stability of CdS is maintained during the post-treatment such as surface modification of CdS or polymerization of PMMA. In order to polymerize the

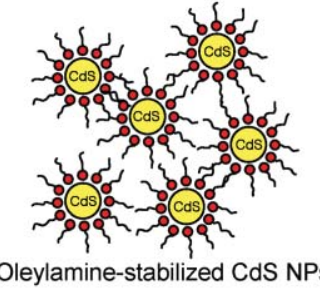

Oleylamine-stabilized CdS NPS

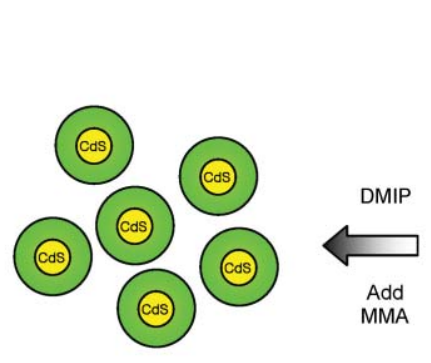

CdS/PMMA core/shell NPs

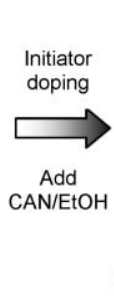

(-) Charge CdS/(+) Charge $\mathrm{Ce}^{4+}$

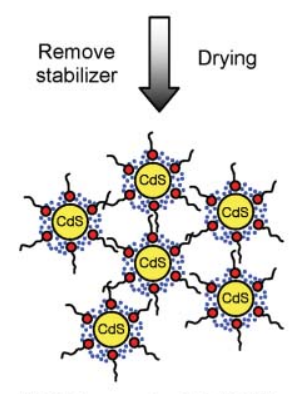

Initiator-coated CdS NPs
Scheme 1 Schematic diagram of the fabrication of CdS/PMMA core/shell nanoparticles. 
MMA monomer on the surface of CdS nanoparticles, CdS nanoparticles were pretreated by ceric ammonium nitrate (CAN). Typically, $0.2 \mathrm{~g}$ of CAN (excess amount with respect to the CdS core) was added into the $\mathrm{CdS} / \mathrm{EtOH}$ solution. The positively charged ceric ion can be attached onto the $\mathrm{CdS}$ nanoparticles by charge-charge interaction because the surface of $\mathrm{CdS}$ has negative charge. ${ }^{9}$ The calculated weight ratio of ceric ion to the CdS NPs is approximately 0.2 . The CAN-coated CdS NPs $(0.1 \mathrm{~g})$ were dispersed in hexane $(30 \mathrm{~mL})$ and then the mixture was heated to $65{ }^{\circ} \mathrm{C}$. The MMA monomer $(1 \mathrm{~mL})$ was injected to the CANcoated CdS/hexane solution. The CAN adsorbed on CdS NPs plays a role in oxidant, and the MMA radical is produced when the medium is heated to $65{ }^{\circ} \mathrm{C} .{ }^{10}$ Therefore, the radical polymerization is initiated from the surface of $\mathrm{CdS}$, and the MMA can be exclusively polymerized on the surfaces of the $\mathrm{CdS}$ NPs.

Fig. 1(a) represents the transmission electron microscopy (TEM) image of spherical CdS NPs synthesized with $\mathrm{CdCl}_{2}$ and sulfur in oleylamine. The CdS NPs were higly monodisperse and their average diameter was $5.4 \mathrm{~nm}$. The TEM image of the CdS/PMMA core/shell NPs is displayed in Fig. 1(b). The diameter of CdS/ PMMA core/shell nanoparticles was $c a .20 \mathrm{~nm}$. It is clearly shown that the CdS/PMMA core/shell nanoparticles maintain their uniform dispersity after removal of capping agent. The magnified TEM image reveals the core/shell nanostructures with the shell thickness of $c a .7 \mathrm{~nm}$. The hydrodynamic radii of CdS NPs and CdS/PMMA core/shell NPs measured by dynamic light scattering were 5.4 and $19.4 \mathrm{~nm}$, respectively, which was in good agreement with the TEM observation. The polydispersity index of the CdS core and CdS/PMMA core/shell nanoparticles were 0.18 and 0.34 , indicating that the particle size distribution was reasonably narrow without serious aggregation. After the polymerization, some disordered shapes of core/shell NPs were observed in the TEM image. During the radical polymerization, MMA monomer might be initiated at each ceric ion site on the CdS surface, and grows randomly in all directions. In a particular case, a propagating MMA chain could recombine with another $\mathrm{Ce}^{4+}$ or MMA radical, and early termination can occur compared with other propagating sites. Therefore, the irregular sphere shape of CdS/PMMA core/ shell species could be formed after the radical polymerization.

Fig. 2 shows the Fourier-transform infrared (FT-IR) spectra of $\mathrm{CdS}$ cores and CdS/PMMA core/shell nanoparticles. The FT-IR spectrum of oleylamine-stabilized CdS nanoparticles revealed the $\mathrm{CH}_{2}$ stretching of oleylamine at 2924 and $2853 \mathrm{~cm}^{-1}$, indicating
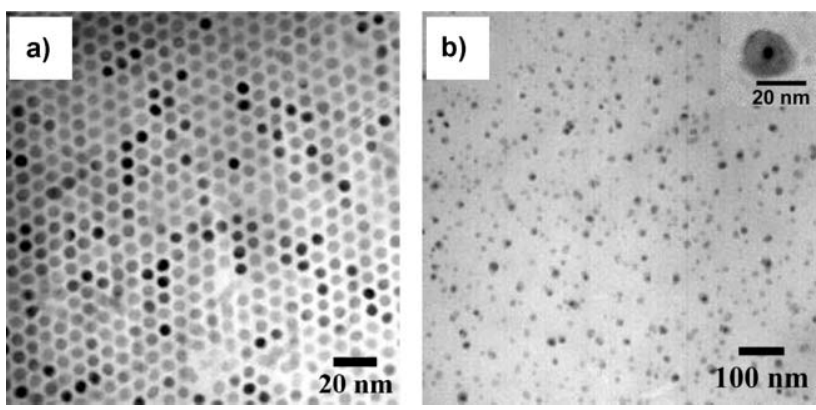

Fig. 1 TEM images of (a) CdS NPs fabricated with $\mathrm{CdCl}_{2}$ and sulfur in oleylamine stabilizer and (b) CdS/PMMA NPs (inset: a magnified TEM image of a single core/shell NP).

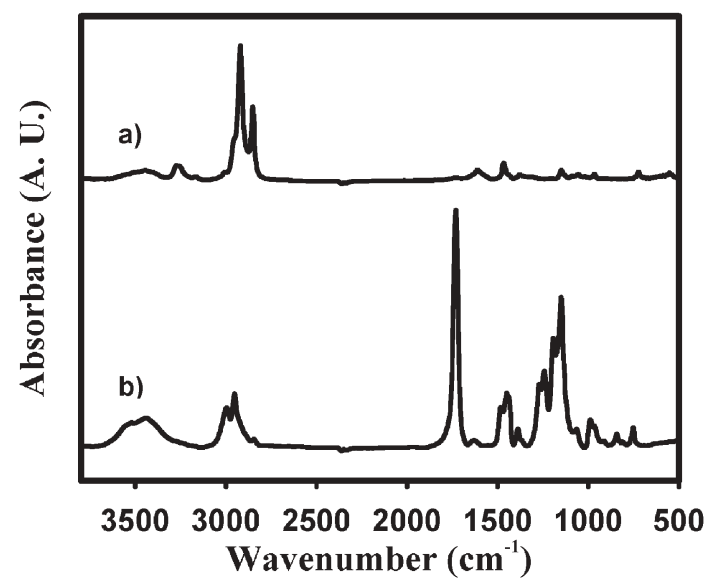

Fig. 2 FT-IR spectra of (a) CdS and (b) CdS/PMMA core/shell NPs.

that the oleylamine still remains after the washing procedure. In the FT-IR spectrum of the CdS/PMMA core/shell nanoparticles, the $\mathrm{C}-\mathrm{O}$ stretching peaks of PMMA appeared at 1150, 1195, 1241 and $1277 \mathrm{~cm}^{-1}$. The peak at $1731 \mathrm{~cm}^{-1}$ related with $\mathrm{C}=\mathrm{O}$ stretching of PMMA was also observed. Moreover, there is no band at $1640 \mathrm{~cm}^{-1}$, which would originate from the double bond of MMA monomer. Judging from these data, it could be confirmed that the PMMA was successfully polymerized on the CdS surface.

UV-Vis absorption and photoluminescence (PL) spectra of CdS NPs are shown in Fig. 3. The UV-Vis of CdS NPs have a maximum absorption peak at $436 \mathrm{~nm}$ (Fig. 3(a)). The PL of sphere-shaped CdS NPs exhibited a sharp peak at $467 \mathrm{~nm}$ (excited at $436 \mathrm{~nm})$, markedly blue shifted $(510 \mathrm{~nm})$ related to that of bulk CdS. ${ }^{11}$ Bulk CdS has been reported to have a broad emission with the emission maximum in the 500-700 nm region of the luminescence spectrum. The quantum yield of $\mathrm{CdS}$ is measured as $c a$. $12 \%$, which is in good agreement with the previous reports. ${ }^{5,12}$ The PL spectrum of CdS/PMMA core/shell NPs is also shown in Fig. 3(c). In the case of CdS/PMMA core/shell NPs, the quantum yield is approximately $8 \%$. While the PL and quantum yield of CdS NPs showed higher intensity than that of CdS/ PMMA core/shell NPs, the emission maximum of the spectra was

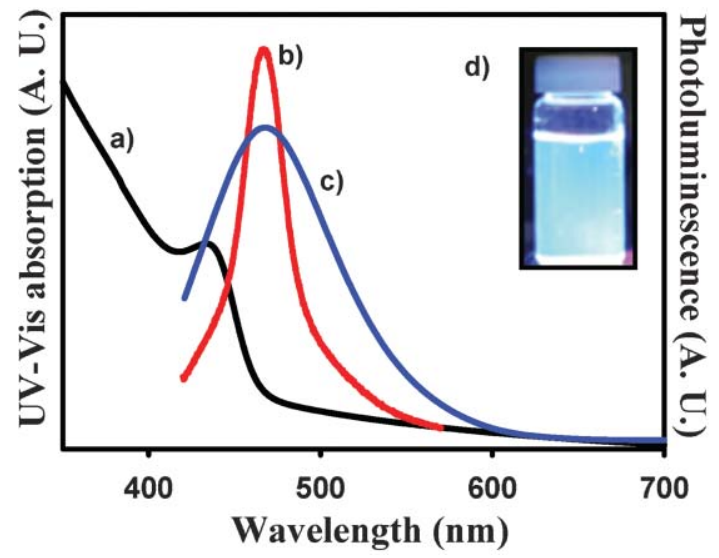

Fig. 3 (a) UV-vis absorption CdS core, (b) PL spectrum of CdS core, (c) PL spectrum of CdS/PMMA core/shell NPs, and (d) emission photograph of CdS/PMMA core/shell NPs. 


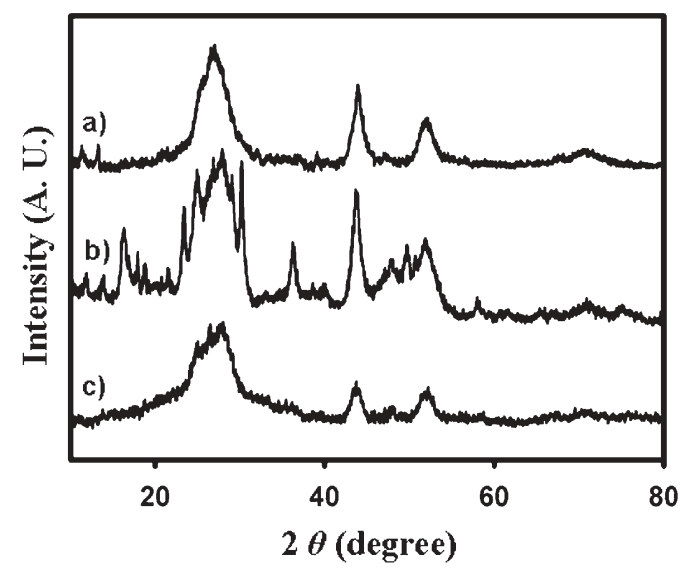

Fig. 4 X-Ray diffraction patterns of (a) as-prepared CdS NPs, (b) CdS NPs after one month, and (c) CdS/PMMA core/shell NPs after one month.

quite similar to each other. This result obviously suggests that the nano-sized PMMA shell around the CdS core does not affect the optical property of the CdS core due to its optical transparency. ${ }^{13}$ Fig. 3(d) shows an emission photograph of CdS/PMMA core/shell NPs. The sample was irradiated by a UV-lamp of $365 \mathrm{~nm}$, and a blue color was observed from CdS NPs, which is well matched with the PL spectra.

Fig. 4 illustrates the X-ray diffraction (XRD) pattern of the asprepared CdS NPs, CdS NPs after one month under air, and CdS/ PMMA core/shell NPs after one month under air. The XRD pattern of as-prepared CdS nanoparticles indicates the characteristic peaks of cubic crystal structure corresponding to (111), (220) and (311) reflections. ${ }^{14}$ As can be seen from Fig. 4(b), the XRD pattern of CdS nanoparticles after one month was different from that of the as-prepared CdS nanoparticles. The CdS nanoparticles had been degraded, so that their original crystal structure was lost. Judging from this result, the surfactant as a stabilizer does not provide CdS nanoparticles with long-term stability and crystallinity under air and against photooxidation. Fig. 4(c) presents the XRD pattern of CdS/PMMA core/shell nanoparticles after one month. The diffraction peaks of Fig. 4(c) were identical with those of Fig. 4(a), which were designated with the cubic crystal system. Therefore, it could be concluded that the CdS nanoparticles were successfully encapsulated by PMMA layers. In addition, the PMMA-coated CdS nanoparticles maintained their surface stability against photooxidation and oxidation under air.

In conclusion, CdS/PMMA core/shell NPs were fabricated using DMIP. The PMMA shell effectively protects the surface of the CdS core from photooxidation and oxidation under air. In addition, the CdS/PMMA core/shell structure maintained the optical properties of the CdS NPs due to the optical transparency of PMMA. Dispersion-mediated interfacial polymerization provided a facile route to prepare the polymer shell on the inorganic core, and can be expanded to the fabrication of various inorganic/ polymer and polymer/polymer core/shell structures.

This work has been financially supported by the Brain Korea 21 program of the Korean Ministry of Education and by Korea
Science and Engineering Foundation through the Hyperstructured Organic Materials Research Center.

\section{Notes and references}

1 C. B. Murray, C. R. Kagan and M. G. Bawendi, Annu. Rev. Mater. Sci., 2000, 30, 545; C. B. Murray, Shouheng Sun, W. Gaschler, H. Doyle, T. A. Betley and C. R. Kagan, J. Res. Dev., 2001, 45, 47; K. Palaniappan, S. A. Hackney and J. Liu, Chem. Commun., 2004, 2704; T. Jin, F. Fujii, H. Sakata, M. Tamura and M. Kinjo, Chem. Commun., 2005, 2829.

2 J. Li and L.-W. Wang, Chem. Mater., 2004, 16, 4012; B. L. Wehrenberg and P. Guyot-Sionnest, J. Am. Chem. Soc., 2003, 125, 7806.

3 J. T. Hu, T. W. Odom and C. M. Lieber, Acc. Chem. Res., 1999, 32, 435; X. Peng, L. Manna, W. Yang, J. Wickham, E. Scher, A. Kadavanich and A. P. Alivisatos, Nature, 2000, 404, 59; I. Robel, B. A. Bunker and P. V. Kamat, Adv. Mater., 2005, 17, 2458; S. Banerjee and S. S. Wong, Chem. Commun., 2004, 1866.

4 V. C. Sundar, H.-J. Eisler and M. G. Bawendi, Adv. Mater., 2002, 14, 739; F. Fleischhaker and R. Zentel, Chem. Mater., 2005, 17, 1346; J.-M. Hsieh, M.-L. Ho, P.-W. Wu, P.-T. Chou, T.-T. Tsai and Y. Chi, Chem. Commun., 2006, 615; G.-P. Wang, E.-Q. Song, H.-Y. Xie, Z.-L. Zhang, Z.-Q. Tian, C. Zuo, D.-W. Pang, D. C. Wu and Y.-B. Shi, Chem. Commun., 2005, 4276; C.-Y. Chen, C.-T. Cheng, C.-W. Lai, P.-W. Wu, K.-C. Wu, P.-T. Chou, Y.-H. Chou and H.-T. Chiu, Chem. Commun., 2006, 263; W. J. Jin, M. T. Fernandez-Arguelles, J. M. CostaFernandez, R. Pereiro and A. Sanz-Medel, Chem. Commun., 2005, 883; L. Dyadyusha, H. Yin, S. Jaiswal, T. Brown, J. J. Baumberg, F. P. Booy and T. Melvin, Chem. Commun., 2005, 3201.

5 J. Joo, H. B. Na, T. Yu, J. H. Yu, Y. W. Kim, F. Wu, J. Z. Zhang and T. Hyeon, J. Am. Chem. Soc., 2003, 125, 11100.

6 T. Yonezawa, S. Onoue and N. Kimizuka, Langmuir, 2001, 17, 2291.

7 G. De and D. Kundu, Chem. Mater., 2001, 13, 4239; D. Holzinger and G. Kickelbick, Chem. Mater., 2003, 15, 4944; J. Yu, J. Yu, Z.-X. Guo and Y.-F. Gao, Macromol. Rapid Commun., 2001, 22, 1261; Y. Li, E. C. Y. Liu, N. Pickett, P. J. Skabara, S. S. Cummins, S. Ryley, A. J. Sutherland and P. O'Brien, J. Mater. Chem., 2005, 15, 1238; D. Wu, X. Ge, Z. Zhang, M. Wang and S. Zhang, Langmuir, 2004, 20, 5192; C.-T. Chen, V. D. Pawar, Y. S. Munot, C.-C. Chen and C.-J. Hsu, Chem. Commun., 2005, 2483.

8 Q. Peng, J. Zhai, W. Wang, X. Yan and F. Bai, Cryst. Growth Des., 2003, 3, 623; A. B. Artyukhin, O. Bakajin, P. Stroeve and A. Noy, Langmuir, 2004, 20, 1442; Y. Tsujii, M. Ejaz, K. Sato, A. Goto and T. Fukuda, Macromolecules, 2001, 34, 8872; K. M. Gattas-Asfura and R. M. Leblane, Chem. Commun., 2003, 2684; B. Pal, T. Torimoto, K. Okazaki and B. Ohtani, Chem. Commun., 2007, 483; J. Jang and B. Lim, Angew. Chem., Int. Ed., 2003, 42, 5600; J. Jang, J. Ha and B. Lim, Chem. Commun., 2006, 1622; H. Duan, M. Kuang, D. Wang, D. G. Kurth and H. Moehwald, Angew. Chem., Int. Ed., 2005, 44, 1717; X. Fan, L. Lin and P. B. Messersmith, Comput. Sci. Technol., 2006, 66, 1198; K. Zhang, H. Li, H. Zhang, S. Zhao, D. Wang and J. Wang, Mater. Chem. Phys., 2006, 96, 477.

9 B. I. I. Shukla, H. Lu, B. Zou, H. Rehage and C. M. Niemeyer, ChemPhysChem, 2006, 7, 1112; G. Guo, W. Liu, J. Liang, Z. He, H. Xu and X. Yang, Mater. Lett., 2007, 61, 1641.

10 G. S. Misra and U. D. N. Bajpai, Prog. Polym. Sci., 1982, 8, 61; A. S. Sarac, Prog. Polym. Sci., 1999, 24, 1149; D. Pramanick and S. K. Sarkar, Colloid Polym. Sci., 1976, 254, 989.

11 C. Klingshirn, Phys. Status Solidi B, 1997, 202, 857; T. Trindade, P. O'Brien and N. L. Pickett, Chem. Mater., 2001, 13, 3843.

12 B. A. Harruff and C. E. Bunker, Langmuir, 2003, 19, 893.

13 A. Lalayan, A. Avetisyan and A. Djotyan, Laser Phys. Lett., 2005, 2, 12; L. Dong, T. Gushtyuk and J. Jiao, J. Phys. Chem. B, 2004, 108, 1617; S. K. Haram, B. M. Quinn and A. J. Bard, J. Am. Chem. Soc., 2001, 123, 8860; K. J. Lee, J. H. Oh, Y. Kim and J. Jang, Adv. Mater., 2006, 18, 2216.

14 Z. H. Zhang, W. S. Chin and J. J. Vittal, J. Phys. Chem. B, 2004, 108, 18569 